\title{
Conjugate Loci of Pseudoriemannian 2-step Nilpotent Lie Groups with Nondegenerate Center
}

\author{
Changrim Jang $^{1}$ and Phillip E. Parker \\ Mathematics Department \\ Wichita State University \\ Wichita KS 67260-0033 \\ USA \\ crjang@math.wichita.edu_phil@math.wichita.edu
}

23 March 2005

\begin{abstract}
We determine the complete conjugate locus along all geodesics parallel or perpendicular to the center (Theorem 2.3). When the center is 1-dimensional we obtain formulas in all cases (Theorem 2.5), and when a certain operator is also diagonalizable these formulas become completely explicit (Corollary 2.7). These yield some new information about the smoothness of the pseudoriemannian conjugate locus. We also obtain the multiplicities of all conjugate points.
\end{abstract}

MSC(2000): Primary 53C50; Secondary 22E25, 53B30, 53C30.

\footnotetext{
${ }^{1}$ On sabbatical leave; permanent address: Department of Mathematics, College of Natural Sciences, University of Ulsan, Ulsan 680-749, Republic of Korea.

Email: crjang@mail.ulsan.ac.kr
} 


\section{Introduction}

Let $N$ denote a nilpotent Lie group with a left-invariant metric tensor. The study of the conjugate locus in these spaces began with O'Sullivan's proof in 1974 that when $N$ is not abelian, there exist conjugate points [9]. In 1981, Naitoh and Sakane showed [7] that some first conjugate points must lie in the center $Z$ of $N$. If $N$ is 2-step nilpotent, they showed that there are no closed geodesics and the cut locus of the identity element $1 \in N$ is nonempty. Finally, when $N$ is the 3-dimensional Heisenberg group $H_{3}$, the cut locus of 1 is precisely the set of first conjugate points to 1 and lies in the center of $H_{3}$. Since then, all results that have been published were for 2 -step nilpotent Lie groups. This class includes the groups of $H$-type; see the introduction of [5] for a brief history of the latter.

Eberlein's 1994 paper [2] sparked a continuing increased interest in 2step nilpotent Lie groups. As he noted, they provide examples in which explicit calculations are frequently feasible and which differ minimally from flat (pseudo-) Euclidean spaces. He obtained only one result on conjugate points, determining conditions under which geodesics tangent to the center contain conjugate points. So far, only Riemannian (positive-definite) metric tensors had been considered. Cordero and Parker [1] extended much of this work to pseudoriemannian (indefinite) metric tensors, including the result on conjugate points; $c f$. Proposition 1.1 infra.

Meanwhile, in 1997, Walschap [11] showed that for a certain class of such Riemannian groups (nonsingular with 1-dimensional center), the cut locus and the conjugate locus coincide, and he made an explicit determination of all first conjugate points in them. Jang and Park [3] later gave explicit formulas for all conjugate points on geodesics either parallel or perpendicular to the center in any Riemannian 2-step nilpotent Lie group, and on all geodesics when the center is 1-dimensional. The present paper extends these results to pseudoriemannian geometries, while providing shorter, simpler, and more conceptual proofs. We also obtain some new information about the smoothness of the conjugate locus which are valid even when there are degenerate [6] conjugate points, which is precisely when the well-known results of Warner [12] do not apply. Indeed, Warner's property (R2) is equivalent to conjugate points being nondegenerate, as is easily seen from his interpretation of it on p. $577 \mathrm{f}$ (and proof on p. 603) of [12].

By an inner product on a vector space $V$ we shall mean a nondegenerate, symmetric bilinear form on $V$, generally denoted by $\langle$,$\rangle . Our convention$ 
is that $v \in V$ is timelike if $\langle v, v\rangle>0$, null if $\langle v, v\rangle=0$, and spacelike if $\langle v, v\rangle<0$.

Throughout, $N$ will denote a connected, simply connected, 2-step nilpotent Lie group with Lie algebra $\mathfrak{n}$ having center $\mathfrak{z}$. This means the Lie group exponential map exp : $\mathfrak{n} \rightarrow N$ is a global diffeomorphism, and we may work in what are usually called "exponential coordinates," making calculations in $\mathfrak{n}$ and taking the result to be in $N$. We shall use $\langle$,$\rangle to denote either$ an inner product on $\mathfrak{n}$ or the induced left-invariant pseudoriemannian (indefinite) metric tensor on $N$. Note that we shall not be using the geodesic (pseudoriemannian) exponential map in this article.

The only general result on conjugate points is Proposition 4.18 from [1]; cf. Proposition 3.10 in Eberlein [2].

Proposition 1.1 Let $N$ be a simply connected, 2-step nilpotent Lie group with left-invariant metric tensor $\langle$,$\rangle , and let \gamma$ be a geodesic with $\dot{\gamma}(0)=$ $a \in \mathfrak{z}$. If $\operatorname{ad}^{\dagger} a=0$, then there are no conjugate points along $\gamma$.

In this paper we make the additional assumption that the center $\mathfrak{z}$ of $\mathfrak{n}$ is nondegenerate. (In the notation of [1], this means $\mathfrak{U}=\mathfrak{V}=\{0\}, \mathfrak{Z}=\mathfrak{z}$, $\mathfrak{E}=\mathfrak{v}=\mathfrak{z}^{\perp}$ and $\mathfrak{n}=\mathfrak{z} \oplus \mathfrak{v}$.)

Theorems 2.3 and 2.5 and Corollary 2.7 generalize and extend the results of [3] to pseudoriemannian spaces. See also [10] for results on the topology of the set of conjugate points along a single geodesic.

Corollaries 2.4 and 2.6 and Proposition 2.8 give some new information on the smoothness of the conjugate locus.

Finally, we also obtain a new formulation of the Jacobi equation for these geometries in Proposition 2.1, of some interest in its own right.

We denote the adjoint with respect to $\langle$,$\rangle of the adjoint representation$ of the Lie algebra $\mathfrak{n}$ on itself by $\operatorname{ad}^{\dagger}$. In the case of a nondegenerate center, the involution $\iota$ of [1] is merely given by $\iota\left(z_{\alpha}\right)=\varepsilon_{\alpha} z_{\alpha}$ and $\iota\left(e_{a}\right)=\bar{\varepsilon}_{a} e_{a}$ where $\left\langle z_{\alpha}, z_{\alpha}\right\rangle=\varepsilon_{\alpha}$ and $\left\langle e_{a}, e_{a}\right\rangle=\bar{\varepsilon}_{a}$ on an orthonormal basis of $\mathfrak{n}$. Then the operator $j: \mathfrak{z} \rightarrow \operatorname{End}(\mathfrak{v})$ is given by $j(z) x=\iota \operatorname{ad}_{x}^{\dagger} \iota z$. We refer to [1] and [8] for other notations and results.

For convenience, we shall use the notation $J_{z}=\operatorname{ad}^{\dagger} z$ for any $z \in \mathfrak{z}$. (The involution $\iota$ does not play much of a role when the center is nondegenerate, and in fact may be omitted.)

We begin by specializing Theorems 3.1 and 3.6 of [1] to the case of a nondegenerate center. 
Proposition 1.2 For all $z, z^{\prime} \in \mathfrak{z}$ and $e, e^{\prime} \in \mathfrak{v}$ we have

$$
\begin{aligned}
\nabla_{z} z^{\prime} & =0, \\
\nabla_{z} e=\nabla_{e} z & =-\frac{1}{2} J_{z} e, \\
\nabla_{e} e^{\prime} & =\frac{1}{2}\left[e, e^{\prime}\right] .
\end{aligned}
$$

Proposition 1.3 For all $z, z^{\prime}, z^{\prime \prime} \in \mathfrak{z}$ and $e, e^{\prime}, e^{\prime \prime} \in \mathfrak{v}$ we have

$$
\begin{aligned}
R\left(z, z^{\prime}\right) z^{\prime \prime} & =0, \\
R\left(z, z^{\prime}\right) e & =\frac{1}{4}\left(J_{z} J_{z^{\prime}} e-J_{z^{\prime}} J_{z} e\right), \\
R(z, e) z^{\prime} & =\frac{1}{4} J_{z} J_{z^{\prime}} e, \\
R(z, e) e^{\prime} & =\frac{1}{4}\left[e, J_{z} e^{\prime}\right], \\
R\left(e, e^{\prime}\right) z & =-\frac{1}{4}\left(\left[e, J_{z} e^{\prime}\right]+\left[J_{z} e, e^{\prime}\right]\right), \\
R\left(e, e^{\prime}\right) e^{\prime \prime} & =\frac{1}{4}\left(J_{\left[e, e^{\prime \prime}\right]} e^{\prime}-J_{\left[e^{\prime}, e^{\prime \prime}\right]} e\right)+\frac{1}{2} J_{\left[e, e^{\prime}\right]} e^{\prime \prime} .
\end{aligned}
$$

To study conjugate points, we shall use the Jacobi operator.

Definition 1.4 Along the geodesic $\gamma$, the Jacobi operator is given by

$$
R_{\dot{\gamma}} \cdot=R(\bullet, \dot{\gamma}) \dot{\gamma}
$$

In physics, this operator measures the relative acceleration produced by tidal forces along $\gamma[8$, p. 219]. For the reader's convenience, we recall that a Jacobi field along $\gamma$ is a vector field along $\gamma$ which is a solution of the Jacobi equation

$$
\nabla_{\dot{\gamma}}^{2} Y(t)+R_{\dot{\gamma}} Y(t)=0
$$

along $\gamma$. The point $\gamma\left(t_{0}\right)$ is conjugate to the point $\gamma(0)$ if and only if there exists a nontrivial Jacobi field $Y$ along $\gamma$ such that $Y(0)=Y\left(t_{0}\right)=0$.

Next, we specialize Proposition 4.8 of [1] to our present setting. As there, $L_{n}$ denotes left translation in $N$ by $n \in N$.

Proposition 1.5 Let $N$ be simply connected, $\gamma$ a geodesic with $\gamma(0)=1 \in$ $N$, and $\dot{\gamma}(0)=z_{0}+x_{0} \in \mathfrak{n}$. Then

$$
\dot{\gamma}(t)=L_{\gamma(t) *}\left(z_{0}+e^{t J} x_{0}\right)
$$

where $J=J_{z_{0}}$. 
As in [3], we shall identify all tangent spaces with $\mathfrak{n}=T_{1} N$. Thus we regard

$$
\dot{\gamma}(t)=z_{0}+e^{t J} x_{0}
$$

as the geodesic equation. Using Proposition 1.3, we compute

Lemma 1.6 The Jacobi operator along the geodesic $\gamma$ in $N$ with $\gamma(0)=1$ and $\dot{\gamma}(0)=z_{0}+x_{0}$ is given by

$$
\begin{aligned}
R_{\dot{\gamma}}(z+x)= & \frac{3}{4} J_{\left[x, x^{\prime}\right]} x^{\prime}+\frac{1}{2} J_{z} J x^{\prime}-\frac{1}{4} J J_{z} x^{\prime}-\frac{1}{4} J^{2} x \\
& -\frac{1}{2}\left[x, J x^{\prime}\right]+\frac{1}{4}\left[x^{\prime}, J x\right]+\frac{1}{4}\left[x^{\prime}, J_{z} x^{\prime}\right]
\end{aligned}
$$

for all $z \in \mathfrak{z}$ and $x \in \mathfrak{v}$, where $x^{\prime}=e^{t J} x_{0}$ and $J=J_{z_{0}}$.

ACKnowledgements: CJ wishes to express his profound gratitude to Prof. K. Park, who may not have been present in corpore but was most definitely present in spiritu during the initial formulation of some proofs. CJ also thanks the Department at Wichita for its hospitality. PEP thanks CJ for his inexhaustible energy and his patience.

\section{Main results}

For later convenience, we separate out the principal part of the necessary computations for Jacobi fields. This yields a new formulation (due to CJ) of the Jacobi equation for these geometries.

Proposition 2.1 Let $\gamma$ be a geodesic with $\gamma(0)=1$ and $\dot{\gamma}(0)=z_{0}+x_{0} \in$ $\mathfrak{z} \oplus \mathfrak{v}=\mathfrak{n}$. A vector field $Y(t)=z(t)+e^{t J} v(t)$ along $\gamma$, where $z(t) \in \mathfrak{z}$ and $v(t) \in \mathfrak{v}$ for each $t$, is a Jacobi field if and only if

$$
\begin{aligned}
\dot{z}(t)-\left[e^{t J} v(t), x^{\prime}(t)\right] & =\zeta, \\
e^{t J} \ddot{v}(t)+e^{t J} J \dot{v}(t)-J_{\zeta} x^{\prime}(t) & =0,
\end{aligned}
$$

where $x^{\prime}(t)=e^{t J} x_{0}$ with $J=J_{z_{0}}$ and $\zeta \in \mathfrak{z}$ is a constant.

Proof: Using Proposition 1.2 and Lemma 1.6, we compute $\nabla_{\dot{\gamma}} Y, \nabla_{\dot{\gamma}}^{2} Y$ and $R_{\dot{\gamma}} Y$ for such a $Y$.

$$
\begin{aligned}
\nabla_{\dot{\gamma}} Y(t)= & \dot{z}(t)-\frac{1}{2} J_{z(t)} x^{\prime}(t)+J e^{t J} v(t)+e^{t J} \dot{v}(t) \\
& -\frac{1}{2} J e^{t J} v(t)+\frac{1}{2}\left[x^{\prime}(t), e^{t J} v(t)\right] \\
= & \dot{z}(t)-\frac{1}{2} J_{z(t)} x^{\prime}(t)+\frac{1}{2} J e^{t J} v(t)+e^{t J} \dot{v}(t)+\frac{1}{2}\left[x^{\prime}(t), e^{t J} v(t)\right],
\end{aligned}
$$




$$
\begin{aligned}
\nabla_{\dot{\gamma}}^{2} Y(t)= & \ddot{z}(t)-\frac{1}{2} J_{\dot{z}(t)} x^{\prime}(t)-\frac{1}{2} J_{\dot{z}(t)} x^{\prime}(t)-\frac{1}{2} J_{z(t)} J x^{\prime}(t) \\
& +\frac{1}{4} J J_{\dot{z}(t)} x^{\prime}(t)-\frac{1}{4}\left[x^{\prime}(t), J_{z(t)} x^{\prime}(t)\right]+\frac{1}{2} J^{2} e^{t J} v(t) \\
& +\frac{1}{2} J e^{t J} \dot{v}(t)-\frac{1}{4} J^{2} e^{t J} v(t)+\frac{1}{4}\left[x^{\prime}(t), J e^{t J} v(t)\right]+J e^{t J} \dot{v}(t) \\
& +e^{t J} \ddot{v}(t)-\frac{1}{2} J e^{t J} \dot{v}(t)+\frac{1}{2}\left[x^{\prime}(t), e^{t J} \dot{v}(t)\right]+\frac{1}{2}\left[J x^{\prime}(t), e^{t J} v(t)\right] \\
& +\frac{1}{2}\left[x^{\prime}(t), J e^{t J} v(t)+e^{t J} \dot{v}(t)\right]-\frac{1}{4} \operatorname{ad}_{x^{\prime}(t)}^{\dagger}\left[x^{\prime}(t), e^{t J} v(t)\right] \\
= & \ddot{z}(t)-J_{\dot{z}(t)} x^{\prime}(t)-\frac{1}{2} J_{z(t)} J x^{\prime}(t) \\
& +\frac{1}{4} J J_{\dot{z}(t)} x^{\prime}(t)-\frac{1}{4}\left[x^{\prime}(t), J_{z(t)} x^{\prime}(t)\right]+\frac{1}{4} J^{2} e^{t J} v(t)+J e^{t J} \dot{v}(t) \\
& +\frac{3}{4}\left[x^{\prime}(t), J e^{t J} v(t)\right]+e^{t J} \ddot{v}(t)+\left[x^{\prime}(t), e^{t J} \dot{v}(t)\right] \\
& +\frac{1}{2}\left[J x^{\prime}(t), e^{t J} v(t)\right]-\frac{1}{4} \mathrm{ad}_{x^{\prime}(t)}^{\dagger}\left[x^{\prime}(t), e^{t J} v(t)\right], \\
R_{\dot{\gamma}} Y(t)= & R_{\dot{\gamma}} z(t)+R_{\dot{\gamma}} e^{t J} v(t) \\
= & \frac{1}{2} J_{z(t)} J x^{\prime}(t)-\frac{1}{4} J J_{z(t)} x^{\prime}(t)+\frac{1}{4}\left[x^{\prime}(t), J_{z(t)} x^{\prime}(t)\right] \\
& +\frac{3}{4} \operatorname{ad}_{x^{\prime}(t)}^{\dagger}\left[e^{t J} v(t), x^{\prime}(t)\right]-\frac{1}{4} J^{2} e^{t J} v(t)-\frac{1}{2}\left[e^{t J} v(t), J x^{\prime}(t)\right] \\
& +\frac{1}{4}\left[x^{\prime}(t), J e^{t J} v(t)\right] \\
&
\end{aligned}
$$

Thus $Y$ is a Jacobi field if and only if

$$
\begin{aligned}
\nabla_{\dot{\gamma}}^{2} Y(t)+R_{\dot{\gamma}} Y(t)= & \ddot{z}(t)-J_{\dot{z}(t)} x^{\prime}(t)+J e^{t J} \dot{v}(t)+\left[x^{\prime}(t), J e^{t J} v(t)\right] \\
& +e^{t J} \ddot{v}(t)+\left[x^{\prime}(t), e^{t J} \dot{v}(t)\right]+\left[J x^{\prime}(t), e^{t J} v(t)\right] \\
& +\operatorname{ad}_{x^{\prime}(t)}^{\dagger}\left[e^{t J} v(t), x^{\prime}(t)\right] \\
= & \frac{d}{d t}\left(\dot{z}(t)-\left[e^{t J} v(t), x^{\prime}(t)\right]\right)+e^{t J} \ddot{v}(t)+e^{t J} J \dot{v}(t) \\
& -\operatorname{ad}_{x^{\prime}(t)}^{\dagger}\left(\dot{z}(t)-\left[e^{t J} v(t), x^{\prime}(t)\right]\right)=0 .
\end{aligned}
$$

Separating this into $\mathfrak{z}$ and $\mathfrak{v}$ components yields the desired result.

Definition 2.2 Let $\gamma$ denote a geodesic and assume that $\gamma\left(t_{0}\right)$ is conjugate to $\gamma(0)$ along $\gamma$. To indicate that the multiplicity of $\gamma\left(t_{0}\right)$ is $m$, we shall write mult $_{c p}\left(t_{0}\right)=m$. To distinguish the notions clearly, we shall denote the multiplicity of $\lambda$ as an eigenvalue of a specified linear transformation by mult $_{e v} \lambda$.

Let $\gamma$ be a geodesic with $\gamma(0)=1$ and $\dot{\gamma}(0)=z_{0}+x_{0} \in \mathfrak{z} \oplus \mathfrak{v}$, respectively, and let $J=J_{z_{0}}$. If $\gamma$ is not null, we may assume $\gamma$ is normalized so that $\langle\dot{\gamma}, \dot{\gamma}\rangle= \pm 1$. As usual, $\mathbb{Z}^{*}$ denotes the set of all integers with 0 removed. We adapt the usual notation from number theory and write $a \mid b$ to denote that $b$ is a nonzero integral multiple of $a$ for real $a, b$. 
Theorem 2.3 Under the preceding assumptions:

1. if $J=0$ and $x_{0}=0$, then there are no conjugate points along $\gamma$;

2. if $J=0$ and $x_{0} \neq 0$, then $\gamma(t)$ is conjugate to $\gamma(0)$ along $\gamma$ if and only if $-12 / t^{2}$ is an eigenvalue of the linear operator

$$
A: \mathfrak{z} \rightarrow \mathfrak{z}: z \mapsto\left[x_{0}, J_{z} x_{0}\right]
$$

and $\operatorname{mult}_{c p}(t)=$ mult $_{e v}\left(-12 / t^{2}\right) \leq \operatorname{dim} \mathfrak{z}$.

3. if $J \neq 0$ and $x_{0}=0$, then $\gamma(t)$ is conjugate to $\gamma(0)$ along $\gamma$ if and only if

$$
t \in \bigcup_{k=1}^{q} \frac{2 \pi}{\lambda_{k}} \mathbb{Z}^{*}
$$

where the $-\lambda_{k}^{2}$ are the negative eigenvalues of $J^{2}$, and

$$
\text { mult }_{c p}\left(\frac{2 \pi}{\lambda_{k}} n\right)=\sum_{\left.\frac{\lambda_{k}}{n}\right|_{\lambda_{h}}} \text { mult }_{e v}\left(-\lambda_{h}^{2}\right) \leq \operatorname{dim} \mathfrak{v} .
$$

If there are no negative eigenvalues in Part 3, then there are of course no conjugate points.

Consider the conjugate points from Part 2 of the preceding theorem for which $z_{0}=0$. Denoting this set by $\mathcal{Z}$, we have $\mathcal{Z} \subseteq \exp \mathfrak{v}$ (the Lie group exponential map). Using standard results from analytic function theory, we immediately obtain the next result.

Corollary 2.4 This part $\mathcal{Z}$ of the conjugate locus is an analytic variety in the submanifold $\exp \mathfrak{v}$ in $N$.

For the convenience of the reader, we sketch one way to prove this using results in the well-known text of Whitney [13]. Complexify. By Theorem V.4A, the mapping of coefficients of the characteristic polynomial of a matrix, thus of the entries, to the eigenvalues is continuous and proper; $c f$. the map $\tau$ on p.355 in item (D), second paragraph. We take $\mathfrak{v}$ as our domain, via the composition of $\tau$ with the map \{components of $x_{0}$ \} $\rightarrow$ $\{$ coefficients of the characteristic polynomial of $A\}$. So we think of the set of eigenvalues as the image of a multifunction of $x_{0} \in \mathfrak{v}$. Now the results in Section 1.7 (pp. 21ff) show that $\tau$ is a holomorphic multifunction, whence so is our composition just defined. Theorems $4.6 \mathrm{~F}, 5.4 \mathrm{~A}$, and/or $7.11 \mathrm{~B}$ yield 
analyticity of the image of (a subset of) the eigenvalues, by the composition of our multifunction with the mapping defined by the geodesic parametrization. (Since $\tau$ is proper, this last composition is at least semiproper, which suffices for the use of Theorem 7.11B.) Finally, our conjugate locus is the real part, thus (real) analytic itself. In the Riemannian case, stronger results follow from Warner [12].

Theorem 2.5 In addition, assume that $\operatorname{dim} \mathfrak{z}=1$. Then:

1. if $z_{0} \neq 0$ and $x_{0}=0$, then $\gamma(t)$ is conjugate to $\gamma(0)$ along $\gamma$ if and only if

$$
t \in \bigcup_{k=1}^{q} \frac{2 \pi}{\lambda_{k}} \mathbb{Z}^{*}
$$

where the $-\lambda_{k}^{2}$ are the negative eigenvalues of $J^{2}$ and

$$
\operatorname{mult}_{c p}\left(\frac{2 \pi}{\lambda_{k}} n\right)=\sum_{\frac{\lambda_{k}}{n} \mid \lambda_{h}} \text { mult }_{e v}\left(-\lambda_{h}^{2}\right) \leq \operatorname{dim} \mathfrak{v} ;
$$

2. if $z_{0}=0$ and $x_{0} \neq 0$, then $\gamma(t)$ is conjugate to $\gamma(0)$ along $\gamma$ if and only if

$$
-\frac{12}{t^{2}}=\varepsilon\left\langle J_{z} x_{0}, J_{z} x_{0}\right\rangle,
$$

where $z \in \mathfrak{z}$ is a unit vector with $\varepsilon=\langle z, z\rangle$, and $\operatorname{mult}_{c p}(t)=1$;

3. if $z_{0} \neq 0$ and $x_{0} \neq 0$, then $\gamma(t)$ is conjugate to $\gamma(0)$ along $\gamma$ if and only if either

$$
t \in \bigcup_{k=1}^{q} \frac{2 \pi}{\lambda_{k}} \mathbb{Z}^{*}
$$

where the $-\lambda_{k}^{2}$ are the negative eigenvalues of $J^{2}$, or $t$ is a solution of

$$
t\left\langle J x_{0},\left(e^{-t J}-I\right)^{-1} x_{0}\right\rangle=\langle\dot{\gamma}, \dot{\gamma}\rangle
$$

in which case $\operatorname{mult}_{c p}(t)=1$. For $t=2 \pi n / \lambda_{k}$, the multiplicities are as follows. If $x_{0} \notin \operatorname{im}\left(e^{-t J}-I\right)$, then

$$
\operatorname{mult}_{c p}(t)=\sum_{\left.\frac{\lambda_{k}}{n}\right|_{\lambda_{h}}} \operatorname{mult}_{e v}\left(-\lambda_{h}^{2}\right)-1 .
$$


If $x_{0} \in \operatorname{im}\left(e^{-t J}-I\right)$, then choose $v$ such that $\left(e^{-t J}-I\right) v=t x_{0}$ and

$$
\text { mult }_{c p}(t)=\left\{\begin{array}{cl}
\sum_{\frac{\lambda_{k}}{n} \mid \lambda_{h}} \text { mult }_{e v}\left(-\lambda_{h}^{2}\right)+1 & \text { if }\left\langle J x_{0}, v\right\rangle=\langle\dot{\gamma}, \dot{\gamma}\rangle, \\
\sum_{\left.\frac{\lambda_{k}}{n}\right|_{\lambda_{h}}} \operatorname{mult}_{e v}\left(-\lambda_{h}^{2}\right) & \text { if }\left\langle J x_{0}, v\right\rangle \neq\langle\dot{\gamma}, \dot{\gamma}\rangle .
\end{array}\right.
$$

As we now assume $\mathfrak{z}$ is 1 -dimensional, Corollary 2.4 simplifies. (Recall that we are using the Lie group exponential map.)

Corollary 2.6 The part $\mathcal{Z}$ of the conjugate locus is an analytic submanifold of the hypersurface exp $\mathfrak{v}$ in $N$.

Alternatively, one could prove this directly from the equation in Part 2.

In the next result, we use the orthogonal direct sum decomposition $\bigoplus_{j=1}^{m} \mathfrak{w}_{j}$ where $J$ leaves each $\mathfrak{w}_{j}$ invariant and each $\mathfrak{w}_{j}$ is an eigenspace of $J^{2}$. Further, $J^{2}$ is negative definite for $1 \leq j \leq q$ and positive definite for $q+1 \leq j \leq m$.

Corollary 2.7 If $J^{2}$ is moreover diagonalizable, then Parts 2 and 3 can be made completely explicit.

2. if $z_{0}=0$ and $x_{0} \neq 0$, then $\gamma(t)$ is conjugate to $\gamma(0)$ along $\gamma$ if and only if

$$
\frac{12}{t^{2}}=\sum_{l=1}^{m-q} \lambda_{q+l}^{2} \varepsilon\left\langle B_{q+l}, B_{q+l}\right\rangle-\sum_{k=1}^{q} \lambda_{k}^{2} \varepsilon\left\langle A_{k}, A_{k}\right\rangle
$$

where $x_{0}=\sum_{k} A_{k}+\sum_{l} B_{q+l}, A_{k} \in \mathfrak{w}_{k}, B_{q+l} \in \mathfrak{w}_{q+l},-\lambda_{k}^{2}$ and $\lambda_{q+l}^{2}$ are the eigenvalues of $J^{2}$ with $J=J_{z}$ for $z \in \mathfrak{z}$ a unit vector, and $\varepsilon=\langle z, z\rangle$;

3. if $z_{0} \neq 0$ and $x_{0} \neq 0$, then $\gamma(t)$ is conjugate to $\gamma(0)$ along $\gamma$ if and only if either

$$
t \in \bigcup_{k=1}^{q} \frac{2 \pi}{\lambda_{k}} \mathbb{Z}^{*}
$$

or $t$ is a solution of

$$
\sum_{k=1}^{q}\left\langle A_{k}, A_{k}\right\rangle \frac{\lambda_{k} t}{2} \cot \frac{\lambda_{k} t}{2}+\sum_{l=1}^{m-q}\left\langle B_{q+l}, B_{q+l}\right\rangle \frac{\lambda_{q+l} t}{2} \operatorname{coth} \frac{\lambda_{q+l} t}{2}=\langle\dot{\gamma}, \dot{\gamma}\rangle
$$

where the $A_{h}, B_{i}$ are as in the preceding part and $\pm \lambda_{j}^{2}$ are the eigenvalues of $J^{2}$ with $J=J_{z_{0}}$ now. 
We finish this section by investigating more closely the smoothness of this conjugate locus near (i.e., in a tubular neighborhood of) the set $\mathcal{Z}$.

Continue with $\operatorname{dim} \mathfrak{z}=1, z \in \mathfrak{z}$ a unit vector with $\langle z, z\rangle=\varepsilon$, and $J_{z}^{2} \operatorname{diag}$ onalizable. Consider $x_{0} \in \mathfrak{v}$ decomposed as $x_{0}=\sum_{k=1}^{p} A_{k}+\sum_{l=p+1}^{m} B_{l}$ where $A_{k}$ and $B_{l}$ are eigenvectors of $J_{z}^{2}$ with corresponding eigenvalues $-\lambda_{k}^{2}, \lambda_{l}^{2}$ where $\lambda_{j}>0$ and $\left\langle x_{0}, x_{0}\right\rangle=\sum_{k}\left\langle A_{k}, A_{k}\right\rangle+\sum_{l}\left\langle B_{l}, B_{l}\right\rangle= \pm 1$ or 0 according as $x_{0}$ is unit timelike, unit spacelike, or null, respectively. Note that the $A_{k}$, $B_{l}$, and eigenvalues depend analytically on $x_{0}$.

We shall study 1-parameter families of geodesics whose initial velocities approach $x_{0}$. So for the parameter $a$ consider the geodesic $\gamma_{a}$ emanating from $1 \in N$ with $\dot{\gamma}_{a}(0)$ the appropriate linear combination of $z$ and $x_{0}$ so that $\gamma_{a}$ is unit speed if $x_{0}$ is nonnull.

\begin{tabular}{c|c|c|c}
$\left\langle x_{0}, x_{0}\right\rangle$ & $\dot{\gamma}_{a}(0)$ & $a$ range & $\left\langle\dot{\gamma}_{a}, \dot{\gamma}_{a}\right\rangle$ \\
\hline$\varepsilon$ & $a z+\sqrt{1-a^{2}} x_{0}$ & $-1 \leq a \leq 1$ & $\varepsilon$ \\
\hline$-\varepsilon$ & $a z+\sqrt{1+a^{2}} x_{0}$ & $a \in \mathbb{R}$ & $-\varepsilon$ \\
\hline 0 & $a z+\sqrt{1+a^{2}} x_{0}$ & $a \in \mathbb{R}$ & $a^{2} \varepsilon$
\end{tabular}

By Corollary 2.7, $\gamma_{a}(t)$ is conjugate to $\gamma_{a}(0)=1$ along $\gamma_{a}$ if $t$ solves, for example,

$$
\begin{aligned}
& \left(1 \mp a^{2}\right) \sum_{k}\left\langle A_{k}, A_{k}\right\rangle \frac{\lambda_{k} a t}{2} \cot \frac{\lambda_{k} a t}{2} \\
+ & \left(1 \mp a^{2}\right) \sum_{l}\left\langle B_{l}, B_{l}\right\rangle \frac{\lambda_{l} a t}{2} \operatorname{coth} \frac{\lambda_{l} a t}{2}=\left\langle\dot{\gamma}_{a}, \dot{\gamma}_{a}\right\rangle
\end{aligned}
$$

for $a \neq 0$, or if $t$ solves

$$
\frac{12}{t^{2}}=\sum_{l} \lambda_{l}^{2} \varepsilon\left\langle B_{l}, B_{l}\right\rangle-\sum_{k} \lambda_{k}^{2} \varepsilon\left\langle A_{k}, A_{k}\right\rangle=\Delta^{2}
$$

for $a=0$. Since there are no real solutions (hence no conjugate points) unless the quantity is positive, we have written it as $\Delta^{2}$ with $\Delta>0$. Note that the left-hand side of (2.1) and $\Delta$ both depend analytically on $x_{0}$.

We claim that for sufficiently small $a$, equation (2.1) has a unique (up to sign), bounded solution $t\left(a, x_{0}\right)$ such that

$$
\lim _{a \rightarrow 0} t\left(a, x_{0}\right)= \pm \frac{2 \sqrt{3}}{\Delta}
$$


analytically in $x_{0}$. We shall only write out the case where $|z|=\left|x_{0}\right|=1$ and $t>0$; the other cases can be handled similarly.

First, note that the functions $x \cot x$ and $x \operatorname{coth} x$ have removable singularities at 0 . Thus we may consider their Maclaurin expansions. To simplify notation, we shall supress explicit $x_{0}$ dependence for a little while.

$$
\begin{aligned}
& \frac{\lambda_{k} a t}{2} \cot \frac{\lambda_{k} a t}{2}=1-\frac{\left(\lambda_{k} a t\right)^{2}}{12}+a^{4} f_{k}(a, t) \\
& \frac{\lambda_{l} a t}{2} \operatorname{coth} \frac{\lambda_{l} a t}{2}=1+\frac{\left(\lambda_{l} a t\right)^{2}}{12}+a^{4} f_{l}(a, t)
\end{aligned}
$$

Here $a^{4} f_{n}(a, t)$ denote the remainders obtained by summing the rest of the series, with $f_{n}(0, t) \neq 0$. Next, substitute these into equation (2.1) with $\left\langle\dot{\gamma}_{a}, \dot{\gamma}_{a}\right\rangle=1$.

$$
\begin{aligned}
f(a, t)= & \left(1-a^{2}\right) \sum_{k}\left\langle A_{k}, A_{k}\right\rangle\left[1-\frac{\left(\lambda_{k} a t\right)^{2}}{12}+a^{4} f_{k}(a, t)\right] \\
& +\left(1-a^{2}\right) \sum_{l}\left\langle B_{l}, B_{l}\right\rangle\left[1+\frac{\left(\lambda_{l} a t\right)^{2}}{12}+a^{4} f_{l}(a, t)\right]-1=0
\end{aligned}
$$

Using $\sum_{k}\left\langle A_{k}, A_{k}\right\rangle+\sum_{l}\left\langle B_{l}, B_{l}\right\rangle=1$, we obtain

$$
a^{2}\left(1-\frac{t^{2}}{12} \Delta^{2}+a^{2} R(a, t)\right)=a^{2} g(a, t)=0,
$$

where $a^{2} R(a, t)$ is the combination of all remaining terms with $R(0, t) \neq 0$.

Observe that $g(0,2 \sqrt{3} / \Delta)=0$ and

$$
\frac{\partial g}{\partial t}\left(0, \frac{2 \sqrt{3}}{\Delta}\right)=-\frac{\Delta}{\sqrt{3}} \neq 0 .
$$

Thus we may apply the Implicit Function Theorem to conclude that, near $(0,2 \sqrt{3} / \Delta)$, there exists a unique, positive, analytic solution $t=t\left(a, x_{0}\right)$. It follows from (2.3) that this $t$ satisfies (2.2). Now we use Corollary 2.6 to conclude the proof of the next result. (Recall that we are using the Lie group exponential map.)

Proposition 2.8 The conjugate locus is smooth (indeed, analytic) across (or through) the hypersurface $\exp \mathfrak{v}$ in $N$. 
Proof: Let $n=\operatorname{dim} N$ and consider $p \in \mathcal{Z}$. Since $\mathcal{Z}$ is smooth, there are $n-2$ smooth coordinate functions centered at $p$ comprising a chart in $\mathcal{Z}$. Now $t=t\left(a, x_{0}\right)$ provides another independent, smooth coordinate function centered at $p$. Together with the $n-2$ we already have, we now have a chart in the conjugate locus centered at $p$. Thus near $\mathcal{Z}$, the conjugate locus is a smooth, codimension-1 submanifold.

Note that this applies only in a (possibly very small) tubular neighborhood of $\mathcal{Z}$ in $N$. Away from $\mathcal{Z}$ (outside this tubular neighborhood), the conjugate locus is merely an analytic variety in $N$. In the Riemannian case, stronger results follow from Warner [12].

\section{$3 \quad$ Proof of Theorem 2.3}

The first part is an immediate consequence of Proposition 1.1 (4.18 in [1]).

For the second part, let $Y(t)=z(t)+e^{t J} v(t)=z(t)+v(t)$ be a nontrivial Jacobi field along $\gamma$ with $Y(0)=Y\left(t_{0}\right)=0$ for some $t_{0} \neq 0$. Then by Proposition 2.1 and $J=0$, we have

$$
\begin{aligned}
\dot{z}(t)-\left[v(t), x_{0}\right] & =\zeta, \\
\ddot{v}(t)-J_{\zeta} x_{0} & =0 .
\end{aligned}
$$

for some constant vector $\zeta \in \mathfrak{z}$. By (3.2) and $v(0)=v\left(t_{0}\right)=0$,

$$
v(t)=\frac{1}{2} t\left(t-t_{0}\right) J_{\zeta} x_{0} .
$$

Substituting this into (3.1), integrating, and using $z(0)=0$ yields

$$
z(t)=\left(\frac{1}{6} t^{3}-\frac{1}{4} t_{0} t^{2}\right)\left[J_{\zeta} x_{0}, x_{0}\right]+t \zeta .
$$

Using $z\left(t_{0}\right)=0$ on (3.4) we find

$$
\left[x_{0}, J_{\zeta} x_{0}\right]=-\frac{12}{t_{0}^{2}} \zeta
$$

If $\zeta=0$, then (3.3) and (3.4) imply that $Y(t)=0$ for all $t$, contradicting the nontriviality of $Y$. Thus $\zeta \neq 0$ whence (3.5) implies that $-12 / t_{0}^{2}$ is an eigenvalue of the linear map $A: \mathfrak{z} \rightarrow \mathfrak{z}: z \mapsto\left[x_{0}, J_{z} x_{0}\right]$. Conversely, if $-12 / t_{0}^{2}$ is an eigenvalue of $A$ there is an eigenvector $\zeta \in \mathfrak{z}$ satisfying (3.5). Then $Y(t)=z(t)+v(t)$, where $z(t)$ and $v(t)$ are given by (3.4) and (3.3), 
respectively, is a nontrivial Jacobi field along $\gamma$ with $Y(0)=Y\left(t_{0}\right)=0$. The multiplicity is obvious.

We turn now to the proof of the third part of Theorem 2.3. Assume that $\gamma\left(t_{0}\right)$ is conjugate to $\gamma(0)$ along $\gamma$. Then there exists a nonzero Jacobi field $Y(t)=z(t)+e^{t J} v(t)$ along $\gamma$ with $Y(0)=Y\left(t_{0}\right)=0$. By Proposition 2.1 and $x_{0}=0$ we have

$$
\begin{aligned}
\ddot{z}(t) & =0, \\
\ddot{v}+J \dot{v} & =0 .
\end{aligned}
$$

From (3.6) and $z(0)=z\left(t_{0}\right)=0$, we see that $z(t)=0$.

Let

$$
p(t)=\prod_{j=1}^{p}\left(t^{2}-2 a_{j} t+\left(a_{j}^{2}+b_{j}^{2}\right)\right)^{k_{j}} \prod_{j=1}^{q}\left(t^{2}+\lambda_{j}^{2}\right)^{l_{j}} \prod_{j=1}^{r}\left(t-\mu_{j}\right)^{m_{j}} \cdot t^{s}
$$

be the characteristic polynomial of $J$ where $a_{j} \neq 0, b_{j} \neq 0, \lambda_{j}>0$, and $\mu_{j} \neq 0$ are all real. Then equation (3.7) can be split into

$$
\begin{aligned}
& \ddot{v}_{1}+J \dot{v}_{1}=0, \\
& \ddot{v}_{2}+J \dot{v}_{2}=0,
\end{aligned}
$$

where $v=v_{1}+v_{2}$ with $\mathfrak{v}_{1} \in \mathfrak{k}$ for

$$
\mathfrak{k}=\operatorname{ker} \prod_{j=1}^{p}\left(J^{2}-2 a_{j} J+\left(a_{j}^{2}+b_{j}^{2}\right) I\right)^{k_{j}} \prod_{j=1}^{q}\left(J^{2}+\lambda_{j}^{2} I\right)^{l_{j}} \prod_{j=1}^{r}\left(J-\mu_{j} I\right)^{m_{j}}
$$

and $v_{2} \in \operatorname{ker} J^{s}$. Applying $J^{s-1}$ to both sides of (3.9), we have $J^{s-1} \ddot{v}_{2}=0$. This and $J^{s-1} v_{2}(0)=J^{s-1} v_{2}\left(t_{0}\right)=0$ imply that $J^{s-1} v_{2}=0$. Next, applying $J^{s-2}$ we have $J^{s-2} \ddot{v}_{2}=0$, which similarly implies that $J^{s-2} v_{2}=0$. Continuing this process, we conclude that $v_{2}=0$. Note that $J$ is invertible on $\mathfrak{k}$, essentially because $\operatorname{ker} J \subseteq \operatorname{ker} J^{s}$; see [4] for some more detailed computations of this type.

Lemma 3.1 Let $A$ be a real nonsingular matrix and $0 \neq t \in \mathbb{R}$. Then $\operatorname{ker}\left(e^{t A}-I\right)=\bigoplus_{t \lambda_{j} \in 2 \pi \mathbb{Z}^{*}} \operatorname{ker}\left(A^{2}+\lambda_{j}^{2} I\right)$ where the $-\lambda_{j}^{2}$ are the negative eigenvalues (if any) of $A^{2}$.

Proof: Let $v \in \operatorname{ker}\left(A^{2}+\lambda_{j}^{2} I\right)$ with $t \lambda_{j} \in 2 \pi \mathbb{Z}^{*}$. Then $e^{t A} v=\left(\cos t \lambda_{j}\right) v+$ $\left(\sin t \lambda_{j}\right) J v / \lambda_{j}=v$ so $\left(e^{t A}-I\right) v=0$ and $v \in \operatorname{ker}\left(e^{t A}-I\right)$.

The converse follows via a straightforward computation using the Jordan canonical form of $A$; $c f$. [4] for similar work. 
Using $v_{1}(0)=0$, from $(3.8)$ we get $v_{1}(t)=\left(e^{-t J}-I\right) v$ for some constant vector $0 \neq v \in \mathfrak{k}$. Since $v_{1}\left(t_{0}\right)=0$, we conclude from this and the lemma that $t_{0} \in \bigcup_{j=1}^{q}\left(2 \pi / \lambda_{j}\right) \mathbb{Z}^{*}$ and

$$
v \in \operatorname{ker}\left(e^{t_{0} J}-I\right) \cap \mathfrak{k}=\bigoplus_{t_{0} \lambda_{j} \in 2 \pi \mathbb{Z}^{*}} \operatorname{ker}\left(J^{2}+\lambda_{j}^{2} I\right) .
$$

Moreover, any Jacobi field vanishing at 0 and $t_{0}$ is easily seen to be of the form

$$
Y(t)=\left(e^{-t J}-I\right) v \quad \text { for some } \quad v \in \bigoplus_{t_{0} \lambda_{j} \in 2 \pi \mathbb{Z}^{*}} \operatorname{ker}\left(J^{2}+\lambda_{j}^{2} I\right),
$$

so the mulitplicity follows by noting that $t_{0} \lambda_{h} \in 2 \pi \mathbb{Z}^{*}$ for $t_{0}=2 \pi n / \lambda_{k}$ is equivalent to $\lambda_{h}=m \lambda_{k} / n$ for some nonzero integer $m$.

\section{Proof of Theorem 2.5}

Again the first part is immediate, this time by the third part of Theorem 2.3. The second part follows from the second part of Theorem 2.3 upon noting that $A$ is now a $1 \times 1$ matrix and that

$$
\left\langle\left[x_{0}, J_{z} x_{0}\right], z\right\rangle=\left\langle J_{z} x_{0}, J_{z} x_{0}\right\rangle .
$$

For the third part, let $Y(t)=\alpha(t) z_{0}+e^{t J} v(t)$ be a Jacobi field along $\gamma$ with $Y(0)=Y\left(t_{0}\right)=0$. Then by Proposition 2.1 we have

$$
\begin{aligned}
\dot{\alpha}(t) z_{0}-\left[e^{t J} v(t), e^{t J} x_{0}\right] & =c z_{0}, \\
e^{t J} \ddot{v}(t)+e^{t J} J \dot{v}(t)-c J e^{t J} x_{0} & =0
\end{aligned}
$$

for a constant $c$. Note that $\left[e^{t J} x, e^{t J} y\right]=[x, y]$ since $\operatorname{dim} \mathfrak{z}=1$, and recall that $J$ commutes with $e^{t J}$. Using these, we simplify the preceding equations as

$$
\begin{aligned}
\dot{\alpha}(t) z_{0}-\left[v(t), x_{0}\right] & =c z_{0}, \\
\ddot{v}+J \dot{v}-c J x_{0} & =0 .
\end{aligned}
$$

Taking the inner product of (4.1) with $z_{0}$ and rearranging,

$$
\dot{\alpha}+\frac{\left\langle J x_{0}, v\right\rangle}{\left\langle z_{0}, z_{0}\right\rangle}=c
$$


and the general solution of (4.2) satisfying $v(0)=0$ is

$$
v(t)=\left(e^{-t J}-I\right) v_{0}+c t x_{0}
$$

for some constant vector $v_{0} \in \mathfrak{v}$.

Assume that $\gamma\left(t_{0}\right)$ is conjugate to $\gamma(0)$ and

$$
t_{0} \in \bigcup_{k=1}^{q} \frac{2 \pi}{\lambda_{k}} \mathbb{Z}^{*} .
$$

We distinguish two cases according as $x_{0}$ is or is not in the image of $e^{-t_{0} J}-I$.

If $x_{0} \notin \operatorname{im}\left(e^{-t_{0} J}-I\right)$, then $v\left(t_{0}\right)=0$ and (4.4) imply $v_{0} \in \operatorname{ker}\left(e^{-t_{0} J}-I\right)$ and $c=0$. Using (4.3) and integrating under $\alpha(0)=0$,

$$
\alpha(t)+\frac{\left\langle J x_{0},(-J)^{-1}\left(e^{-t J}-I\right) v_{0}-t v_{0}\right\rangle}{\left\langle z_{0}, z_{0}\right\rangle}=0 .
$$

Since $\alpha\left(t_{0}\right)=0$, it follows that $\left\langle J x_{0}, v_{0}\right\rangle=0$. Thus if $Y$ is a Jacobi field along $\gamma$ such that $Y(0)=Y\left(t_{0}\right)=0$, then (4.5) implies that

$$
v(t)=\left(e^{-t J}-I\right) v_{0},
$$

for some $v_{0} \in \operatorname{ker}\left(e^{-t_{0} J}-I\right)$ with $\left\langle J x_{0}, v_{0}\right\rangle=0$, and

$$
\alpha(t)=\frac{\left\langle J x_{0}, t v_{0}-(-J)^{-1}\left(e^{-t J}-I\right) v_{0}\right\rangle}{\left\langle z_{0}, z_{0}\right\rangle} .
$$

Lemma 4.1 For $x \in \mathfrak{v},\langle x, v\rangle=0$ for all $v \in \operatorname{ker}\left(e^{-t_{0} J}-I\right)$ if and only if $x \in \operatorname{im}\left(e^{-t_{0} J}-I\right)$.

We omit the proof, which is a reasonably straightforward computation in linear algebra. One does need to note that $x_{0} \notin \operatorname{im}\left(e^{-t_{0} J}-I\right)$ if and only if $J x_{0} \notin \operatorname{im}\left(e^{-t_{0} J}-I\right)$, to use the orthogonal decomposition $\mathfrak{v}=$ $\left(\bigoplus_{t_{0} \lambda_{i} \in 2 \pi \mathbb{Z}} \operatorname{ker}\left(J^{2}+\lambda_{i}^{2}\right)^{l_{i}}\right) \oplus \mathfrak{v}^{\prime}$ where $\mathfrak{v}^{\prime}$ is a $J$-invariant, nondegenerate subspace of $\mathfrak{v}$ on which $\left(e^{-t_{0} J}-I\right)$ is invertible, and to use an appropriate block-diagonal representation of $J$ on subspaces of the kernel summands. See, for example, [4] for several similar such computations.

This lemma now yields mult ${ }_{c p}\left(t_{0}\right)=\operatorname{dim} \operatorname{ker}\left(e^{-t_{0} J}-I\right)-1$ and the multiplicity formula follows as previously. 
If $x_{0} \in \operatorname{im}\left(e^{-t_{0} J}-I\right)$, then we can take $v_{1} \in \mathfrak{v}$ such that $\left(e^{-t_{0} J}-I\right) v_{1}=$ $t_{0} x_{0}$. Using (4.4) and $v\left(t_{0}\right)=0$, we obtain $v_{0}=v_{2}-c v_{1}$ for some $v_{2} \in$ $\operatorname{ker}\left(e^{-t_{0} J}-I\right)$. From (4.3), integrating under $\alpha(0)=0$ yields

$$
\alpha(t)+\frac{\left\langle J x_{0},(-J)^{-1}\left(e^{-t J}-I\right) v_{0}-t v_{0}\right\rangle}{\left\langle z_{0}, z_{0}\right\rangle}=c t .
$$

Using $\alpha\left(t_{0}\right)=0$, Lemma 4.1, and the skewadjointness of $J$, a short computation shows

$$
c\left(\left\langle J x_{0}, v_{1}\right\rangle-\langle\dot{\gamma}, \dot{\gamma}\rangle\right)=0 .
$$

By Lemma 4.1, $\left\langle J x_{0}, v_{1}\right\rangle$ is independent of the choice of such a $v_{1}$. We distinguish two subcases according as $\left\langle J x_{0}, v_{1}\right\rangle$ is or is not equal to $\langle\dot{\gamma}, \dot{\gamma}\rangle$.

If $\left\langle J x_{0}, v_{1}\right\rangle=\langle\dot{\gamma}, \dot{\gamma}\rangle$, then we have

$$
\begin{gathered}
v(t)=\left(e^{-t J}-I\right)\left(v_{2}-c v_{1}\right)+c t_{0} x_{0}, \\
\alpha(t)=c t-\frac{\left\langle J x_{0},(-J)^{-1}\left(e^{-t J}-I\right)\left(v_{2}-c v_{1}\right)+t c v_{1}\right\rangle}{\left\langle z_{0}, z_{0}\right\rangle}
\end{gathered}
$$

for $v_{2} \in \operatorname{ker}\left(e^{-t_{0} J}-I\right)$ and arbitrary scalar $c$, whence

$$
\operatorname{mult}_{c p}\left(t_{0}\right)=\operatorname{dim} \operatorname{ker}\left(e^{-t_{0} J}-I\right)+1
$$

and the multiplicity formula follows as before.

If $\left\langle J x_{0}, v_{1}\right\rangle \neq\langle\dot{\gamma}, \dot{\gamma}\rangle$, then (4.6) implies $c=0$ and we find

$$
v(t)=\left(e^{-t J}-I\right) v_{0}
$$

for some $v_{0} \in \operatorname{ker}\left(e^{-t_{0} J}-I\right)$,

$$
\alpha(t)=-\frac{\left\langle x_{0},\left(e^{-t J}-I\right) v_{0}\right\rangle}{\left\langle z_{0}, z_{0}\right\rangle}
$$

and $\operatorname{mult}_{c p}\left(t_{0}\right)=\operatorname{dim} \operatorname{ker}\left(e^{-t_{0} J}-I\right)$, from which the desired formula follows as before.

Now assume that $\gamma\left(t_{0}\right)$ is conjugate to $\gamma(0)$ and

$$
t_{0} \notin \bigcup_{k=1}^{q} \frac{2 \pi}{\lambda_{k}} \mathbb{Z}^{*} .
$$


If $c=0$, then $v\left(t_{0}\right)=0$ implies $\left(e^{-t_{0} J}-I\right) v_{0}=0$ in (4.4). But then (4.7) and Lemma 3.1 forces $v_{0}=0$ in (4.4). Thus $u=0$ and $\left\langle J x_{0}, u\right\rangle=0$, so together with (4.3) and $\alpha(0)=\alpha\left(t_{0}\right)=0$ these imply that $\alpha=0$. Consequently, $Y=0$ which contradicts our assumption that $Y$ is a nontrivial Jacobi field. Therefore $c \neq 0$.

Using (4.4) and $v\left(t_{0}\right)=0$, we get

$$
v(t)=c t x_{0}-\left(e^{-t J}-I\right)\left(e^{-t_{0} J}-I\right)^{-1} c t_{0} x_{0} .
$$

Substituting this into (4.3), we find

$$
\dot{\alpha}(t)-\frac{\left\langle J x_{0},\left(e^{-t J}-I\right)\left(e^{-t_{0} J}-I\right)^{-1} c t_{0} x_{0}\right\rangle}{\left\langle z_{0}, z_{0}\right\rangle}=c .
$$

Integrating with $\alpha(0)=0$ yields

$$
\begin{aligned}
& \alpha(t)=c t \\
& \quad+\frac{\left\langle J x_{0},(-J)^{-1}\left(e^{-t J}-I\right)\left(e^{-t_{0} J}-I\right)^{-1} c t_{0} x_{0}-t\left(e^{-t_{0} J}-I\right)^{-1} c t_{0} x_{0}\right\rangle}{\left\langle z_{0}, z_{0}\right\rangle} .
\end{aligned}
$$

Since $\alpha\left(t_{0}\right)=0$, from (4.9) we obtain

$$
0=1+\frac{\left\langle J x_{0},(-J)^{-1} x_{0}-t_{0}\left(e^{-t_{0} J}-I\right)^{-1} x_{0}\right\rangle}{\left\langle z_{0}, z_{0}\right\rangle} .
$$

Thus

$$
0=-\left\langle z_{0}, z_{0}\right\rangle-\left\langle x_{0}, x_{0}\right\rangle+t_{0}\left\langle J x_{0},\left(e^{-t_{0} J}-I\right)^{-1} x_{0}\right\rangle
$$

or

$$
\begin{aligned}
\langle\dot{\gamma}, \dot{\gamma}\rangle & =\left\langle z_{0}, z_{0}\right\rangle+\left\langle x_{0}, x_{0}\right\rangle \\
& =t_{0}\left\langle J x_{0},\left(e^{-t_{0} J}-I\right)^{-1} x_{0}\right\rangle .
\end{aligned}
$$

Therefore, if $\gamma\left(t_{0}\right)$ is conjugate to $\gamma(0)$ along $\gamma$ and (4.7) holds, then $t_{0}$ is a solution of

$$
t\left\langle J x_{0},\left(e^{-t J}-I\right)^{-1} x_{0}\right\rangle=\langle\dot{\gamma}, \dot{\gamma}\rangle .
$$

The multiplicity follows from the fact that $v$ in (4.8) and $\alpha$ in (4.9) are uniquely determined by the scalar $c$. 


\section{$5 \quad$ Proof of Corollary 2.7}

For Part 2, let $z$ be a unit vector in $\mathfrak{z}$ and consider the inner product $\left\langle\left[x_{0}, J_{z} x_{0}\right], z\right\rangle$. In this part of the proof $J$ denotes $J_{z}$. Thus,

$$
\begin{aligned}
\left\langle\left[x_{0}, J x_{0}\right], z\right\rangle & =\left\langle J x_{0}, J x_{0}\right\rangle \\
& =\left\langle-J^{2} x_{0}, x_{0}\right\rangle .
\end{aligned}
$$

Since $J^{2}$ is nonsingular and diagonalizable, $\mathfrak{v}=\bigoplus_{k=1}^{q} \mathfrak{w}_{k} \oplus \bigoplus_{l=1}^{m-q} \mathfrak{w}_{q+l}$ where $\mathfrak{w}_{k}$ is the eigenspace of $-\lambda_{k}^{2}$ and $\mathfrak{w}_{q+l}$ is the eigenspace of $\lambda_{q+l}^{2}$. Writing $x_{0}=\sum_{k=1}^{q} A_{k}+\sum_{l=1}^{m-q} B_{q+l}$ with $A_{k} \in \mathfrak{w}_{k}$ and $B_{q+l} \in \mathfrak{w}_{q+l}$, we have

$$
\begin{aligned}
\left\langle\left[x_{0}, J x_{0}\right], z\right\rangle & =\left\langle-J^{2} x_{0}, x_{0}\right\rangle \\
& =\sum_{k=1}^{q} \lambda_{k}^{2}\left\langle A_{k}, A_{k}\right\rangle-\sum_{l=1}^{m-q} \lambda_{q+l}^{2}\left\langle B_{q+l}, B_{q+l}\right\rangle .
\end{aligned}
$$

This implies that

$$
\left[x_{0}, J x_{0}\right]=\varepsilon\left(\sum_{k=1}^{q} \lambda_{k}^{2}\left\langle A_{k}, A_{k}\right\rangle-\sum_{l=1}^{m-q} \lambda_{q+l}^{2}\left\langle B_{q+l}, B_{q+l}\right\rangle\right) z
$$

where $\varepsilon=\langle z, z\rangle$. By the second part of Theorem 2.5, $\gamma\left(t_{0}\right)$ is conjugate to $\gamma(0)$ along $\gamma$ if and only if

$$
-\frac{12}{t_{0}^{2}}=\varepsilon\left(\sum_{k=1}^{q} \lambda_{k}^{2}\left\langle A_{k}, A_{k}\right\rangle-\sum_{l=1}^{m-q} \lambda_{q+l}^{2}\left\langle B_{q+l}, B_{q+l}\right\rangle\right) .
$$

Finally, we give the proof of Part 3 of Corollary 2.7. Now we have $\dot{\gamma}(t)=z_{0}+e^{t J} x_{0}$ with $z_{0} \neq 0 \neq x_{0}$ and $J=J_{z_{0}}$ again, as usual. We shall continue with the notations for eigenvalues, eigenspaces, and components of $x_{0}$ as in the preceding Part 2.

It will suffice to show that

$$
\begin{aligned}
& t\left\langle J x_{0},\left(e^{-t J}-I\right)^{-1} x_{0}\right\rangle= \\
& \sum_{k=1}^{q}\left\langle A_{k}, A_{k}\right\rangle \frac{\lambda_{k} t}{2} \cot \frac{\lambda_{k} t}{2}+\sum_{l=1}^{m-q}\left\langle B_{q+l}, B_{q+l}\right\rangle \frac{\lambda_{q+l} t}{2} \operatorname{coth} \frac{\lambda_{q+l} t}{2} .
\end{aligned}
$$

Using the $J$-invariance of each $\mathfrak{w}_{j}$ and the orthogonality of the direct sum decomposition, we have

$$
\begin{aligned}
& t\left\langle J x_{0},\left(e^{-t J}-I\right)^{-1} x_{0}\right\rangle= \\
& t \sum_{k=1}^{q}\left\langle J A_{k},\left(e^{-t J}-I\right)^{-1} A_{k}\right\rangle+t \sum_{l=1}^{m-q}\left\langle J B_{q+l},\left(e^{-t J}-I\right)^{-1} B_{q+l}\right\rangle .
\end{aligned}
$$


If $A_{k} \neq 0$, then $\llbracket A_{k}, \lambda_{k}^{-1} J A_{k} \rrbracket$ is a 2-dimensional complement in $\mathfrak{w}_{k}$ of a $J$-invariant subspace $\mathfrak{w}_{k}^{\prime}$. On this planar subspace, the matrix of $e^{-t J}-I$ is

$$
\left[\begin{array}{cc}
\cos t \lambda_{k}-1 & \sin t \lambda_{k} \\
-\sin t \lambda_{k} & \cos t \lambda_{k}-1
\end{array}\right]
$$

so the matrix of $\left(e^{-t J}-I\right)^{-1}$ is

$$
\left[\begin{array}{cc}
-\frac{1}{2} \csc ^{2}\left(t \lambda_{k} / 2\right) & -\frac{1}{2} \cot \left(t \lambda_{k} / 2\right) \\
\frac{1}{2} \cot \left(t \lambda_{k} / 2\right) & -\frac{1}{2} \csc ^{2}\left(t \lambda_{k} / 2\right)
\end{array}\right]
$$

and it follows that

$$
\begin{aligned}
\left\langle J A_{k},\left(e^{-t J}-I\right)^{-1} A_{k}\right\rangle & =\left\langle J A_{k},-\frac{1}{2} \csc ^{2} \frac{t \lambda_{k}}{2} A_{k}+\frac{1}{2} \cot \frac{t \lambda_{k}}{2} \frac{J A_{k}}{\lambda_{k}}\right\rangle \\
& =\frac{\lambda_{k}}{2} \cot \frac{t \lambda_{k}}{2}\left\langle A_{k}, A_{k}\right\rangle
\end{aligned}
$$

Now assume $J B_{q+l} \neq \pm \lambda_{q+l} B_{q+l}$. Then $B_{q+l}$ and $J B_{q+l}$ are linearly independent, so we may consider the plane $\llbracket \lambda_{q+l}^{-1} J B_{q+l}+B_{q+l}, \lambda_{q+l}^{-1} J B_{q+l}-$ $B_{q+l} \rrbracket$ in $\mathfrak{w}_{q+l}$. Here the matrix of $e^{t J}-I$ is

$$
\left[\begin{array}{cc}
e^{-t \lambda_{q+l}}-1 & 0 \\
0 & e^{t \lambda_{q+l}}-1
\end{array}\right]
$$

so the matrix of $\left(e^{-t J}-I\right)^{-1}$ is

$$
\left[\begin{array}{cc}
\frac{1}{e^{-t \lambda_{q}+l}-1} & 0 \\
0 & \frac{1}{e^{t \lambda_{q+l}}-1}
\end{array}\right]
$$

and we obtain

$$
\begin{aligned}
& \left(e^{-t J}-I\right)^{-1} B_{q+l}= \\
& \quad \frac{\frac{1}{2}}{e^{-t \lambda_{q+l}}-1}\left(\frac{1}{\lambda_{q+l}} J B_{q+l}+B_{q+l}\right)-\frac{\frac{1}{2}}{e^{t \lambda_{q+l}}-1}\left(\frac{1}{\lambda_{q+l}} J B_{q+l}-B_{q+l}\right)
\end{aligned}
$$

whence

$$
\begin{aligned}
\left\langle J B_{q+l},\left(e^{-t J}-I\right)^{-1} B_{q+l}\right\rangle & =\frac{\lambda_{q+l}}{2}\left\langle B_{q+l}, B_{q+l}\right\rangle\left(\frac{1}{e^{t \lambda_{q+l}}-1}-\frac{1}{e^{-t \lambda_{q+l}}-1}\right) \\
& =\frac{\lambda_{q+l}}{2}\left\langle B_{q+l}, B_{q+l}\right\rangle \operatorname{coth} \frac{t \lambda_{q+l}}{2} .
\end{aligned}
$$


Finally, assume $J B_{q+l}= \pm \lambda_{q+l} B_{q+l}$. Then

$$
\begin{aligned}
\left(e^{-t J}-I\right) B_{q+l} & =\left(e^{\mp t \lambda_{q+l}}-1\right) B_{q+l}, \\
\left(e^{-t J}-I\right)^{-1} B_{q+l} & =\frac{1}{e^{\mp t \lambda_{q+l}}-1} B_{q+l} .
\end{aligned}
$$

Using $\left\langle J B_{q+l}, B_{q+l}\right\rangle=0$, it follows much as before that

$$
\left\langle J B_{q+l},\left(e^{-t J}-I\right)^{-1} B_{q+l}\right\rangle=\frac{\lambda_{q+l}}{2}\left\langle B_{q+l}, B_{q+l}\right\rangle \operatorname{coth} \frac{t \lambda_{q+l}}{2} .
$$

Combining (5.1), (5.2), and (5.3) we obtain

$$
\begin{aligned}
& t\left\langle J x_{0},\left(e^{-t J}-I\right)^{-1} x_{0}\right\rangle \\
& =t \sum_{k=1}^{q}\left\langle J A_{k},\left(e^{-t J}-I\right)^{-1} A_{k}\right\rangle+t \sum_{l=1}^{m-q}\left\langle J B_{q+l},\left(e^{-t J}-I\right)^{-1} B_{q+l}\right\rangle \\
& =\sum_{k=1}^{q}\left\langle A_{k}, A_{k}\right\rangle \frac{t \lambda_{k}}{2} \cot \frac{t \lambda_{k}}{2}+\sum_{l=1}^{m-q}\left\langle B_{q+l}, B_{q+l}\right\rangle \frac{t \lambda_{q+l}}{2} \operatorname{coth} \frac{t \lambda_{q+l}}{2}
\end{aligned}
$$

as desired.

\section{References}

[1] L. A. Cordero and P. E. Parker, Pseudoriemannian 2-step nilpotent Lie groups, Santiago-Wichita preprint DGS/CP4, 1999.

arXiv: math.DG/9905188

[2] P. Eberlein, Geometry of 2-step nilpotent groups with a left invariant metric, Ann. scient. Éc. Norm. Sup. 27 (1994) 611-660.

[3] C. Jang and K. Park, Conjugate points on 2-step nilpotent groups, Geom. Dedicata 79 (2000) 65-80.

[4] C. Jang and P.E. Parker, Skewadjoint operators on pseudoeuclidean spaces, Wichita-Ulsan preprint DGS/JP2, 2003.

arXiv: math.DG/0302030

[5] C. Jang, P.E. Parker and K. Park, PseudoH-type 2-step nilpotent Lie groups, Wichita-Ulsan preprint DGS/JP4, 2003. To appear in Houston J. Math. arXiv: math.DG/0307368 
[6] F. Mercuri, P. Piccione, and D.V. Tausk, Stability of the conjugate index, degenerate conjugate points and the Maslov index in semiRiemannian geometry, Pacific J. Math. 206 (2002) 375-400.

arXiv: math.DG/9905096

[7] H. Naitoh and Y. Sakane, On conjugate points of a nilpotent Lie group, Tsukuba J. Math. 5 (1981) 143-152.

[8] B. O’Neill, Semi-Riemannian Geometry. New York: Academic Press, 1983.

[9] J. J. O'Sullivan, Manifolds without conjugate points, Math. Ann. 210 (1974) 295-311.

[10] P. Piccione and D. V. Tausk, On the distribution of conjugate points along semi-Riemannian geodesics, Comm. Anal. Geom. 11 (2003) 33-48. arXiv: math.DG/0011038

[11] G. Walschap, Cut and conjugate loci in two-step nilpotent Lie groups, J. Geometric Anal. 7 (1997) 343-355.

[12] F. W. Warner, The conjugate locus of a Riemannian manifold, Am. J. Math. 87 (1965) 575-604.

[13] H. Whitney, Complex Analytic Varieties. Reading: Addison-Wesley, 1972. 